\title{
Off-pump surgery does not reduce stroke, compared with results of on- pump coronary artery bypass grafting: A meta-analysis of randomized clinical trials
}

Hisato Takagi, MD, PhD, Toshiyuki Tanabashi, MD, Norikazu Kawai, MD, and Takuya Umemoto, MD, PhD, Shizuoka, Japan

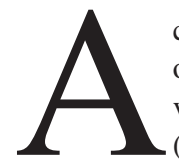

ccording to a meta-analysis by Cheng and associates ${ }^{1}$ of 21 randomized clinical trials (RCTs) of off-pump versus on-pump coronary artery bypass grafting (CABG), postoperative 30-day stroke was not statistically significantly reduced (odds ratio, $0.68 ; 95 \%$ confidence interval [CI], 0.33-1.40) in the off-pump group. In the most recent meta-analysis by Sedrakyan and colleagues ${ }^{2}$ of 16 RCTs, however, off-pump use was associated with $50 \%$ relative risk (RR) reduction of stroke (RR, 0.50; 95\% CI, 0.27-0.93) as compared with that with on-pump CABG. To determine whether off-pump surgery is associated with reduced occurrence of stroke as compared with that of on-pump CABG, we performed a meta-analysis of currently available RCTs of off-pump versus on-pump CABG and discussed the discordant results found in the published literature.

\section{Materials and Methods}

All RCTs in which stroke was reported, comparing results of off-pump versus on-pump CABG, were identified using a 2-level search strategy. First, a public domain database (MEDLINE) was searched using a Web-based search engine (PubMed). Second, relevant studies were identified through a manual search of secondary sources including references of initially identified articles and a search of reviews and commentaries. The MEDLINE database was searched from January 1966 to March 2007. MeSH keywords included "coronary artery bypass, off-pump," "cerebrovascular accident," and "randomized controlled trials." Studies considered for inclusion met the following criteria: the design was an RCT; patients were randomly assigned to off-pump versus on-pump CABG; and main outcomes included stroke. Data regarding detailed inclusion criteria, duration of follow-up, and stroke were abstracted (as available) from each individual study. The criteria for stroke included descriptions such as "stroke," "severe neurological deficit," "cerebrovascular accident," "cerebral embo-

From the Department of Cardiovascular Surgery, Shizuoka Medical Center, Shizuoka, Japan.

Received for publication June 17, 2007; revisions received July 7, 2007; accepted for publication July 10, 2007.

Address for reprints: Hisato Takagi, MD, PhD, Department of Cardiovascular Surgery, Shizuoka Medical Center, 762-1 Nagasawa, Shimizu-cho, Sunto-gun, Shizuoka 411-8611, Japan (E-mail: kfgth973@ybb.ne.jp).

J Thorac Cardiovasc Surg 2007;134:1059-60

$0022-5223 / \$ 32.00$

Copyright () 2007 by The American Association for Thoracic Surgery

doi:10.1016/j.jtcvs.2007.07.006 lism," and "cerebral infarct." We conducted a meta-analysis of summary statistics from the individual trials because detailed, patient-level data were not available for all trials. For each study, data regarding stroke in both the off-pump and on-pump groups were used to generate RRs $(<1$, favors off-pump CABG; $>1$, favors on-pump CABG) and 95\% CIs. Study-specific estimates were combined using inverse variance-weighted averages of logarithmic RRs in a random-effects model. Between-study heterogeneity was analyzed by standard $\chi^{2}$ tests, with $P<.05$ deemed statistically significant. Publication bias was assessed graphically by a funnel plot and mathematically by an adjusted rank-correlation test.

\section{Results}

Our search identified 32 RCTs, in which postoperative 30-day (or in-hospital) stroke was reported, of off-pump versus on-pump CABG (Table 1). We excluded trials reporting more than 30-day stroke. Pooled analysis of all the 32 trials (representing 3714 patients) demonstrated a statistically nonsignificant reduction in stroke with off-pump relative to on-pump CABG $(0.49 \%$ vs $1.29 \%$; RR, 0.60; 95\% CI, 0.34-1.06). There was neither trial heterogeneity of results nor evidence of significant publication bias. When data from 18 trials (representing 2607 patients) with at least one event in either the off-pump or on-pump group were pooled, off-pump CABG was associated with a statistically significant $50 \%$ reduction in stroke relative to that of on-pump CABG (0.69\% vs $1.85 \%$; RR, $0.50 ; 95 \%$ CI, 0.26-0.98). There was neither trial heterogeneity of results nor evidence of significant publication bias.

\section{Discussion}

The limitations of the previous meta-analysis by Sedrakyan and coworkers $^{2}$ are the inclusion of 9 trials reporting postoperative more than 30-day stroke and the exclusion of 11 trials with no event in both the off-pump and on-pump groups. In consequence, the meta-analysis included 16 trials with at least one event in either the off-pump or on-pump group from all the 27 trials reporting stroke, and only 7 of the included 16 trials reported postoperative 30-day stroke. Following the example of the meta-analysis by Cheng and associates, ${ }^{1}$ the present metaanalysis included currently available all the 32 trials reporting postoperative 30-day stroke, and 14 trials of them reported no event in both the off-pump and on-pump groups. Pooled analysis of all the RCTs demonstrated a statistically nonsignificant reduction in stroke with off-pump relative to on-pump CABG. Subgroup analysis eliminating RCTs with no event in both the 
TABLE 1. Outcomes in the trials

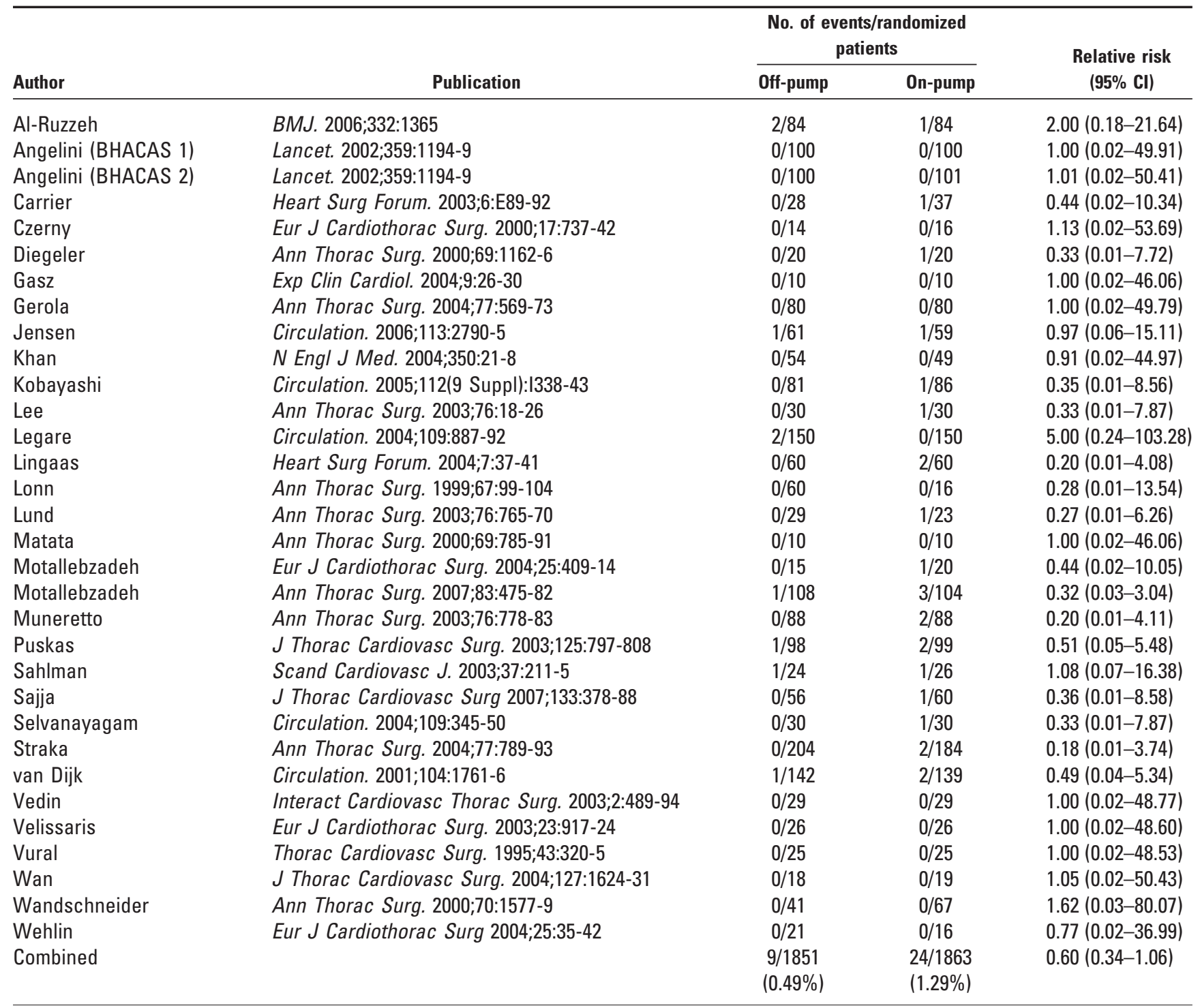

$\mathrm{Cl}$, Confidence interval.

off-pump and on-pump groups demonstrated a statistically significant reduction in stroke. However, this finding should be interpreted with caution, because approximately $30 \%$ of patients were excluded: 1107 (in the 14 trials with no event in both the off-pump and on-pump groups) of 3714 (in all the 32 trials). Because RCTs with no event in both the groups do demonstrate equal risk of the event, eliminating them may mislead the pooled point estimate into being significant decrease or increase of the risk.

\section{References}

1. Cheng DC, Bainbridge D, Martin JE, Novick RJ, Evidence-Based Perioperative Clinical Outcomes Research Group. Does off-pump coronary artery bypass reduce mortality, morbidity, and resource utilization when compared with conventional coronary artery bypass? A metaanalysis of randomized trials. Anesthesiology. 2005;102:188-203.

2. Sedrakyan A, Wu AW, Parashar A, Bass EB, Treasure T. Off-pump surgery is associated with reduced occurrence of stroke and other morbidity as compared with traditional coronary artery bypass grafting: a metaanalysis of systematically reviewed trials. Stroke. 2006;37:2759-69. 